\title{
México en el mundo de las telecomunicaciones: más allá de Slim y la ocDE
}

Mexico in the telecommunications world: Slim and beyond OECD

\section{Sergio Ordóñez, Rafael Bouchaín, Gustavo Schinca*}

Investigadores del Instituto

de Investigaciones Económicas

y de la Facultad de Ciencias de la UNAM

$<<$ serorgu@gmail.com $>>$

$k<$ modeloinsumoproducto@gmail.com $>>$ $<<$ schinca@ciencias.unam.mx $>>$

Journal Economic Literature (JEL):

L96, L41, L51

Palabras clave:

Telecomunicaciones

Monopolización

Economía de la regulción

Keywords:

Telecommunications

Monopolization

Economics of Regulation

\section{Resumen}

En este trabajo se lleva a cabo un análisis comparativo internacional de la situación y grado de desarrollo de la industria de servicios de telecomunicaciones en México (ISTC) en relación con sesenta y cuatro países, entre desarrollados, en desarrollo y directamente competidores de México en la exportación de servicios intensivos en conocimiento que requieren de una ISTC competitiva, a partir del uso de componentes principales como herramienta estadística. El análisis se lleva a cabo en el marco de la airada polémica entre Slim y la OCDE suscitada por la publicación en enero de 2012 del informe denominado "Estudio de la OCDE sobre políticas y regulación de telecomunicaciones en México"; polémica que está en línea de continuidad con la orientación general de la actual iniciativa de reforma de las telecomunicaciones. Se busca proporcionar indicadores internacionales amplios -más que los de la OCDE- para entender tanto la perspectiva de Slim como la de la OCDE en la polémica, así como la orientación general de la actual reforma, bajo la consideración de que la solución de la problemática de la ISTC requiere de horizontes mucho más amplios a los planteados hasta ahora, para propiciar el tránsito de la industria desde su modalidad de desarrollo actual rentista-monopólica a una basada en la innovación de los servicios y la competitividad.

\section{Abstract}

By using Principal Components as statistical tool, this paper deals with an international comparative analysis of the situation and development stage of the telecommunication services industry in Mexico (TLSI) in comparison with 64 countries, including developed, developing and direct Mexico's competitors in knowledge intensive services exportations, which requires a competitive TLSI. This analysis is made within the context of Slim's and OECD's vigorous debate provoked by the January 2012 report called OECD Review of Telecommunication Policy and Regulation in Mexico. Thus, the debate is in line of continuity with the recent telecommunication reform initiative general orientation. We look forward providing wide international indicators -wider than the OECD's- so as to understand Slim's and OECD's perspective in the debate, as well as the recent reform general orientation, under the consideration that the TLSI problematic solution requires much wider analyses horizons than those evoked until now, so as to promote the industry transit from it's actual monopolistic-rentist way of development to a new one based on service innovation and competitiveness.

*Con la colaboración de Benjamín Lujano, investigación realizada gracias al programa UNAM-DGA PA-PAPIIT IN306910. 


\section{Introducción}

El 30 de enero de 2012, la Organización para la Cooperación y el Desarrollo Económicos (OCDE) publicó el informe "Estudio de la OCDE sobre políticas y regulación de telecomunicaciones en México", el cual arrojaba dos importantes conclusiones sobre la situación de la industria de servicios de telecomunicaciones (ISTC) en el país: la existencia de una empresa dominante que inhibe la competencia y de un sistema jurídico ineficiente, con una institución oficial regulatoria débil, los cuales terminan siendo los mayores obstáculos para lograr una industria competitiva que genere mejores servicios para sus usuarios. ${ }^{2}$

Posteriormente y tras calificar de "absurdos, refritos y mentirosos" los resultados del informe, el Director General de América Móvil, envió una carta el 18 de mayo de 2012 al Secretario General de la OCDE, solicitando que "retire inmediatamente su informe" por utilizar métodos deficientes y llegar a conclusiones falsas. ${ }^{3}$

En este trabajo se lleva a cabo un análisis comparativo internacional de la situación de la ISTC en México en relación con otros 63 países, entre desarrollados, en desarrollo y directamente competidores de México en la exportación de servicios intensivos en conocimiento que requieren de una ISTC competitiva, a partir del uso de componentes principales como herramienta estadística. Se busca proporcionar indicadores internacionales amplios -más que los de la OCDE- para entender tanto la perspectiva de Slim como la de la OCDE en la polémica, bajo la consideración de que la solución de la problemática de la ISTC requiere de horizontes mucho más amplios a los planteados por ambos.

Para ello se ha organizado la exposición en tres apartados: en el primero se ubica la importancia de la ISTC en el capitalismo del conocimiento como nueva fase de desarrollo; en el segundo se lleva a cabo el análisis comparativo internacional de la situación de la ISTC en México tanto desde la perspectiva de Slim como de la OCDE; para en el último concluir con el planteamiento de la perspectiva que posibilite el pasaje a una ISTC competitiva basada en la innovación de los servicios.

\section{Capitalismo del conocimiento e ISTC}

Si bien la incorporación del conocimiento en la producción material es un proceso consustancial a la humanidad desde sus orígenes (y que distingue al hombre del resto de los animales), tal proceso da un salto de calidad en el capitalismo, gracias a la conversión

2 "El desempeño insatisfactorio de la industria de telecomunicaciones en México es consecuencia de la inflexible conducta del operador incumbente de telefonía fija y móvil (Telmex y Telcel) con considerable poder de mercado, así como de un sistema jurídico disfuncional que promueve una industria ineficiente, que no es atractiva para la formación de alianzas internacionales y, por lo mismo, resulta dañina para el potencial económico del país" (op. cit, p.123).

${ }^{3}$ Para refutar el estudio, Carlos Slim pagó dos análisis: el primero titulado "Corrigiendo la valoración errónea de la OCDE sobre la competencia de las telecomunicaciones en México" elaborado por Jerry Hausman, director del programa de Investigación en Economía de las Telecomunicaciones del Massachusetts Institute of Technology (MIT), y Agustin Ros, vicepresidente de NERA Economic Consulting; y un segundo estudio titulado "La propuesta de la OCDE por cartelizar las telecomunicaciones mexicanas" elaborado por Gregory Sidak, presidente de Criterion Economics, consultoría que agrupa a prestigiados especialistas de universidades como Columbia, Princeton, Stanford y Yale. 
de la ciencia en fuerza productiva como lo constatara Marx en su momento. Pero lo que resulta crucial en la actualidad es que en el seno de este modo de producción se está verificando un nuevo e inusitado salto de calidad en el proceso, que implica una extensión e imbricación del ciclo del conocimiento (producción, circulación y acumulación) en todos los ámbitos de la reproducción económica y social, para dar paso a una nueva fase de desarrollo o capitalismo de conocimiento. ${ }^{4}$

Por consiguiente, el elemento distintivo más importante en la nueva fase de desarrollo de la economía mundial es la conversión del conocimiento en la principal fuerza productiva, lo que se traduce en un incremento notable del contenido en conocimiento de la producción social a partir de los años ochenta del siglo anterior (Ordóñez, 2004).

El capitalismo del conocimiento nace entonces de una nueva articulación entre el sector científico-educativo (SC-E) y el conjunto de la producción social, lo que se expresa, por ejemplo, en el incremento del número de artículos científicos citados en las patentes concedidas por la United States Patent and Trademark Office (USPTO) en Estados Unidos, donde el promedio se incrementa de 0.5 a 3 de 1987 a 1998, observándose un incremento similar en las patentes concedidas en otros países

el capitalismo del conocimiento nace de una nueva articulación entre el sector científico-educativo y el conjunto de la producción social

\section{desarrollados. ${ }^{5}$}

Adicionalmente, el conocimiento tiene una importancia fundamental en la nueva revolución tecnológica de la informática y las telecomunicaciones, ${ }^{6}$ al posibilitar el surgimiento de una nueva fuerza productiva a partir del estrechamiento del vínculo entre ciencia y conocimiento con la producción social, básicamente mediante dos procesos:

1. El incremento de la capacidad de procesamiento de la información y la producción de ciencia y conocimiento de manera accesible y aplicable a la producción, que resulta, respectivamente, del desarrollo del microprocesador y del software;

2. El incremento en la velocidad y la escala de alcance y difusión del conocimiento y la información, resultado de la confluencia de la informática y las telecomunicaciones, y del desarrollo de éstas.

El despliegue de la revolución informática y de las telecomunicaciones trae consigo la integración de un nuevo complejo tecnológico -productivo, constituido por el conjunto de actividades industriales y de servicios articulados por las tecnologías básicas del circuito integrado, el software y la digitalización o sector electrónico- informático y de telecomunicaciones (SE-IT).?

${ }^{4}$ Una fase de desarrollo del capitalismo se establece cuando una revolución tecnológica se traduce en una nueva base productiva y una nueva forma de producción, lo que trae consigo el surgimiento de nuevos productos, servicios y ramas de actividad, los cuales se convierten en los sectores que tienden a articular al resto de la actividad económica y dinamizar el crecimiento (Ordóñez y Bouchain, 2011).

${ }^{5}$ Como Canadá de 0.5 a 2.5, Suecia de 0.5 a 1.5 y Francia de 0.5 a 1 (Ordóñez y Bouchain, 2011).

${ }^{6}$ Se entiende por revolución tecnológica a un conjunto de innovaciones "incrementales" (de continuidad en una misma base tecnológica), radicales (de ruptura con ella) y que puede abarcar a un conjunto de nuevos sistemas tecnológicos con repercusiones directas o indirectas en casi todas las ramas de actividad, es decir, un cambio en lo que Freeman y Pérez [1998] llaman paradigma tecnoeconómico.

${ }^{7}$ Generalmente se denomina al sector como industrias de tecnologías de la información y comuni- 
El SE-IT se convierte en el nuevo núcleo articulador y dinamizador de la producción, el crecimiento y el comercio mundiales, en sustitución del complejo automotriz-metalmecánico-petroquímico, propio de la anterior fase de desarrollo fordista-keynesiana, lo que se traduce en una nueva dinámica económica o ciclo industrial, con fases expansivas más largas y de mayor crecimiento y fases recesivas más breves y menos profundas.

El SE-IT dinamiza y articula entonces la fase expansiva de los años noventa del siglo anterior, constituye el epicentro de la crisis mundial 2001-2002, y encabeza la recuperación posterior, a partir de un proceso de reestructuración tecnológico-productiva con consecuencias en el despliegue espacial mundial y su división internacional e interindustrial del trabajo (Dabat y Ordóñez, 2009). Lo anterior dará lugar a nuevas tendencias de desarrollo a partir de la cuales el sector vuelve a dinamizar y articular el crecimiento en la recuperación actual (Ordóñez y Bouchaín, 2011).

El SE-IT constituye un complejo industrial y de servicios particularmente integrado, como resultado de las grandes interacciones y sinergias derivadas del sistema tecnológico común que articula sus partes, ${ }^{8}$ y básicamente se encuentra estructurado en cinco grandes actividades productivas: industria electrónica, software y servicios de computación, servicios de telecomunicaciones, producción y distribución de contenidos en medios masivos, actividades de comercialización, distribución y alquiler (Ordóñez, Bouchain, 2011:38 -42).

La industria de servicio de telecomunicaciones (ISTC) es, por tanto, un componente fundamental del SE-IT que desempeña un papel crucial en el desarrollo de los países en las nuevas condiciones, al construir la infraestructura de telecomunicaciones tanto para el desarrollo del ciclo interno del conocimiento en las economías nacionales, como para la integración internacional de éstas en los procesos globales, en el marco de una nueva división global del trabajo basada cada vez más en el conocimiento. En esa perspectiva, la ISTC se convierte en una condición infraestructural fundamental para la exportación de servicios intensivos en conocimiento por medio de procesos de relocalización-subcontratación internacionales, en los cuales recientemente un conjunto de países latinoamericanos, donde México figura sólo marginalmente, se ha incorporado importantemente a partir del aprovechamiento de ventajas competitivas en relación con los competidores del sudeste asiático (véase Ordóñez, prensa). ${ }^{9}$

cación (TIC), denominación utilizada por instituciones como el Departamento de Comercio de Estados Unidos, la OCDE o el Foro Económico Mundial (WEF), y adoptado por muchos autores, la cual tiene el inconveniente de apelar a las tecnologías en las que se basa el sector -sin ser suficientemente riguroso en este sentido, dado que las tecnologías básicas son el circuito integrado, el software y la digitalización-, y no a la naturaleza de los productos y servicios que provee, criterio a partir del cual se propone la denominación de SE-IT (Ordóñez, Bouchain, 2011:37).

${ }^{8}$ Existen diversos ejemplos de esa interrelación: la electrónica de consumo y las telecomunicaciones tomaron de la computadora las tecnologías de control y software, al tiempo que le aportaban los métodos de producción a gran escala común a ambas, las capacidades gráfica y de almacenamiento óptico propias de la electrónica de consumo y las telecomunicaciones.

${ }^{9}$ Los principales países que se incorporan en este proceso (por orden decreciente en su monto exportador) son: Brasil, Argentina, México, Chile, Costa Rica, Colombia y Uruguay. Los servicios de mayor dinamismo para la región son (en orden decreciente), los servicios de computación e informática, servicios de licencias y derechos, servicios empresariales, servicios financieros, servicios de seguros, servicios personales, culturales y recreativos y servicios de telecomunicaciones. Véase Ordóñez, en prensa. 


\section{México en el mundo de las telecomunicaciones: análisis comparativo mediante componentes principales}

mediante un análisis basado en componentes principales (CP) por ser una técnica estadística que se usa para reducir la dimensión de un conjunto extenso de datos. En nuestro caso se trata de realizar una tipología sobre el desarrollo de las ISTC en 64 países seleccionados a partir de 30 variables elegidas de la base de datos proporcionada por la Unión Internacional de Telecomunicaciones en su informe de $2010,{ }^{10}$ y ubicar a México en ese contexto.

Se seleccionaron un conjunto de variables que reflejaran el desarrollo relativo de las ISTC, de acuerdo con los siguientes parámetros: 1) los niveles de penetración de los servicios (6 variables); 2) 18 tarifas relacionadas con la telefonía fija y celular, además de una adicional que representa el costo del internet de banda ancha reportado por Netindex en megabytes por segundo, ${ }^{11}$ medidas tanto en dólares corrientes como en dólares en PPP (Purchasing Power Parity) ligados a Estados Unidos, lo cual resulta pertinente debido a que la paridad real ${ }^{12}$ de las monedas oscila en torno a su poder de compra comparativo, y éste constituye, en consecuencia, un parámetro adecuado para comparar los precios internos de los servicios entre los países y la capacidad de sus sociedades de acceder a los servicios de la ISTC; ${ }^{13}$ 3) 2 variables relativas a los niveles de inversión e ingresos de las telecomunicaciones, 4) 3 variables que se refieren a la capacidad de integración al mercado mundial, mediante la exportación de servicios intensivos en conocimiento y que requieren de una ISTC competitiva, de acuerdo con lo planteado más arriba. Para una descripción general del uso de $\mathrm{CP}$, véase el anexo metodológico.

a) Nivel de cobertura (penetración), inversión y capacidad de integración comparativas mundiales de la ISTC en México

México se encuentra entre las ISTC más rezagadas de los 64 países analizados, pues aparece en el extremo derecho de la gráfica $1 .{ }^{14}$ Cercanos a México se observan cinco

${ }^{10}$ La UIT es el organismo de las Naciones Unidas que recopila y publica alrededor de 160 variables del sector telecomunicaciones para 212 países, el reporte de 2010 contiene series que van de 1960 a 2009. Debido a la restricción en la disponibilidad de los datos reportados por los países, y en virtud de que el análisis de CP requiere información completa, nuestro universo se redujo a 29 variables para 64 países. Otra variable fue extraída de netindex. Véase anexo metodológico.

${ }^{11}$ Las 29 variables provenientes de la uाT se colectaron en 90\% para el año de 2008, el resto corresponde a información reportada entre 2005 y 2007. Además se consideró como indicador más preciso del precio de acceso a internet al precio del megabyte por segundo en lugar de la renta mensual del servicio, reportado por la ITU.

${ }^{12}$ De acuerdo con la teoría del valor-trabajo, la paridad real del tipo de cambio es la paridad resultante del poder adquisitivo comparativo de las monedas, en torno a la cual se establece la paridad de mercado en la que influye el flujo de divisas sobre una economía, resultado del comercio internacional y la inversión extranjera (directa y de cartera).

${ }^{13}$ En el caso de México, la medición en dólares PPP significa eliminar la sobrevaluación acumulada 2000-2008 de 4.51\% del peso frente al dólar, la cual hace aparecer los precios internos medidos en dólares corrientes como si fueran más reducidos en ese porcentaje (durante el mismo periodo), lo que ilusoriamente mejora la posición competitiva de la ISTC del país.

${ }^{14}$ En las gráficas que a continuación se presentan, la longitud de las flechas indica el grado de incidencia relativa de la variable correspondiente en la localización de los países (véase anexo metodológico). 
países del sureste asiático que son sus competidores en el mercado mundial de servicios intensivos en conocimiento y basados en telecomunicaciones: India, Tailandia, Indonesia, Vietnam y Filipinas (Ordóñez y Bouchaín, 2011). Se aprecia también que estos cinco países se encuentran en la mitad inferior de la Gráfica, lo cual indica algunas diferencias importantes con México que aparece más arriba, ya que en este país la cobertura en telefonía fija (19\%) y en internet $(8 \%)$ es superior a la existente en India (3 y $1 \%$ respectivamente), Indonesia (13 y 1\%), Filipinas (5 y 4\%) y en Tailandia (11 y 3\%) pero, por otra parte, la cobertura en telefonía celular en los casos de Tailandia (91\%), Vietnam (86\%) y Filipinas (75\%), es mayor que la que hay en México (69\%) y también existen mayores niveles de inversión en los casos de India (58\%), Filipinas (45\%) e Indonesia (37\%), respecto a México (27\%).

\section{Gráfica 1 \\ Cobertura, inversión y capacidad de integración internacional}

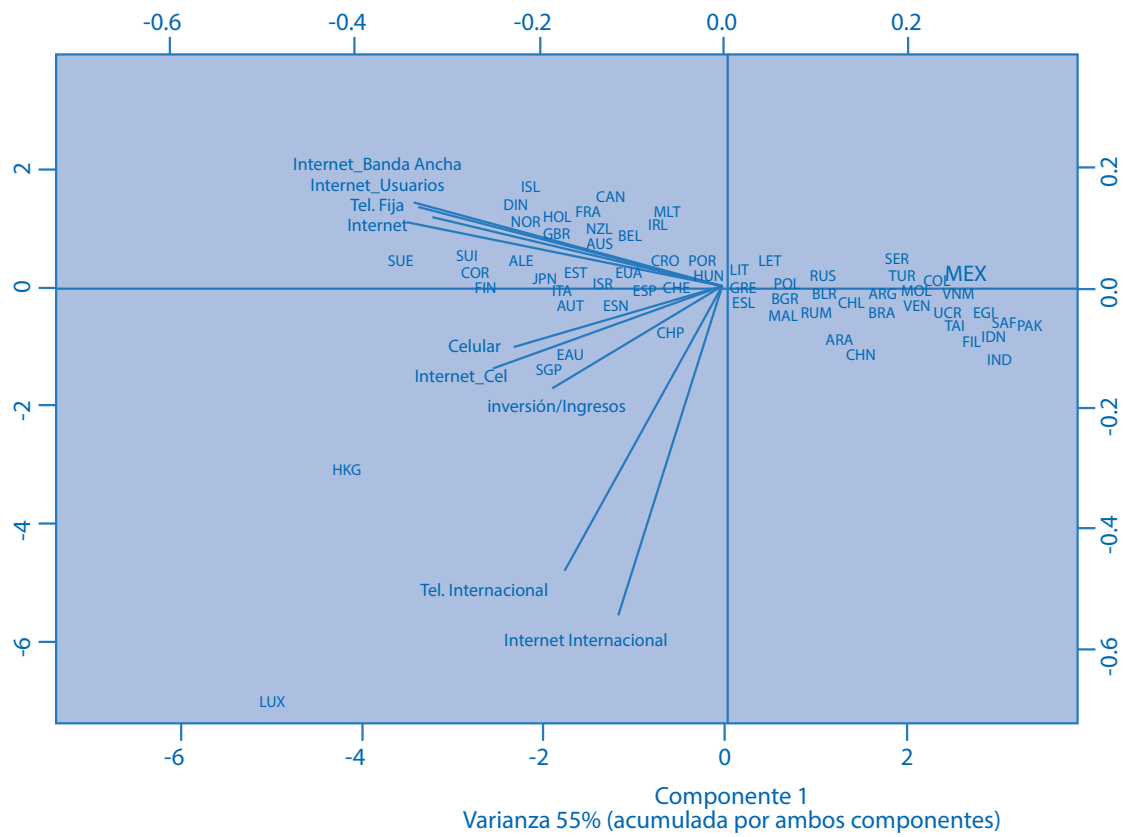

Cabe destacar que Malasia es el país con la ISTC menos rezagada en el sureste asiático en cuanto cobertura y capacidad de integración al mercado mundial de servicios basados en la ISTC, pues se encuentra cerca del centro de la gráfica junto con varios países de Europa oriental como Rusia, Bielorrusia, Polonia, Eslovaquia, Bulgaria, Rumania y Grecia. Es importante mencionar que México es el país con el mayor déficit en tráfico telefónico internacional ${ }^{15}$ de los 64 países observados con 81 minutos anualmente por habitante, lo cual también tiende a posicionarlo más arriba en la gráfica.

Por otra parte, en la mitad del lado izquierdo se encuentran los países con ISTC más desarrolladas, entre los que se encuentran 28 de los 34 países miembros de la OCDE, de los

${ }^{15}$ En realidad el dato reportado en la balanza de pagos por el FMl en el rubro de servicios de telecomunicaciones sería un mejor indicador en este aspecto, pero no fue posible utilizarlo debido a que no se encuentra suficientemente desagregado para conocer el dato correspondiente específicamente a la telefonía fija. 
cuales cabe destacar a Corea, Estonia, Eslovenia, Hungría, República Checa, Irlanda, Portugal, España, Italia e Israel, en tanto que podrían servir como modelo de desarrollo para la ISTC en nuestro país. En este mismo sector izquierdo se encuentran otros seis países que no son miembros de la OCDE y que también podrían servir como referencia para el desarrollo de la ISTC en México, como son Hong Kong, Singapur, Emiratos Árabes, Chipre, Malta y Croacia.

De los países de la OCDE, México es uno de los seis con mayor rezago en su ISTC además de Chile, Turquía, Grecia, Eslovaquia y Polonia, lo que se traduce en que todos estos se encuentran en la mitad del lado derecho de la gráfica. Aun cuando Grecia, Eslovaquia y Polonia se hallan muy cerca del centro, lo cual indica un menor rezago en sus ISTC.

En cuanto a países latinoamericanos, se aprecia que Chile es el país con la ISTC menos rezagada en esta región en cuanto a cobertura y capacidad de integración internacional pues se encuentra un poco más a la izquierda que Argentina, Brasil, Venezuela, Colombia y México. Esto se debe a que en Chile hay una mayor proporción de internautas (39\%) y a su mayor capacidad de tráfico internacional de internet $(0.004 \mathrm{Mb} / \mathrm{s}$ por habitante) respecto a los otros países. También se observa que México aparece más arriba que los demás países latinoamericanos debido, por una parte, a que la cobertura en telefonía celular $(69 \%)$ es la más baja en este conjunto de países -en los cuales hay alrededor de noventa líneas de telefonía celular por cada 100 habitantes-, y, por la otra, a que en México se tiene el mayor déficit en tráfico telefónico internacional de los 64 países. ${ }^{16}$

En los casos de China y Arabia Saudita, sus ISTC están menos rezagadas que en los países del sureste asiático (excepto Malasia) y su posición en la parte inferior se debe a que China tiene la tasa más alta de inversión (63\%) en telecomunicaciones de los 64 países considerados y al uso muy intensivo de la telefonía celular y de la telefonía fija internacional en el caso de Arabia Saudita (143\% y 48 minutos anualmente por habitante).

En cuanto a los países europeos, Turquía, Serbia, Ucrania y Moldavia se encuentran muy rezagados en cuanto a cobertura y capacidad de integración internacional al igual que los países latinoamericanos y del sureste asiático (excepto Malasia).

Finalmente, los dos países africanos incluidos en el análisis, Egipto y Sudáfrica, se encuentran en el mismo sector que los países más rezagados del sureste asiático, lo cual indica que sus ISTC tienen niveles de cobertura y de capacidad de integración internacional equiparables al de esos países.

b) Mediciones de precios en dólares corrientes vs poder adquisitivo real de los usuarios (PPP)

En el mundo de Slim México aparece en el centro de la gráfica 2, lo cual indica que en el país los precios de las telecomunicaciones medidos en dólares corrientes son en conjunto más bajos que en los 33 países que aparecen más a la izquierda en la gráfica, entre los cuales se encuentran 24 países europeos, además de Estados Unidos, Canadá, Brasil, Chile, Venezuela, Japón, Australia, Nueva Zelanda y Sudáfrica. Por otra parte, los precios

${ }^{16}$ Es muy probable que en ello incidan muy significativamente las llamadas entrantes que realiza la población emigrante y residente en Estados Unidos. En cuanto a los países miembros de la OCDE, la posición de México en la gráfica 1 coincide en gran medida con los resultados reportados el pasado mes de enero (http://dx.doi.org/10.1787/9789264166790-es) en sus gráficas que van de la 1.1 a la 1.6 en donde nuestro país aparece como uno de los que tienen penetración más baja de telefonía fija, de telefonía móvil y de banda ancha. 
de las telecomunicaciones así medidos son en conjunto más altos respecto a los 30 países que aparecen a la derecha, entre los cuales destacan casi la totalidad de los quince países asiáticos incluidos en el análisis, que se encuentran mucho más a la derecha, lo

en México se tiene que indica que en estos países los precios de las telecomunicaciones en conel mayor déficit junto son mucho más bajos que en México. Japón es el único país asiático en en tráfico telefónico donde los precios de las telecomunicaciones en conjunto son más altos que en internacional México, por lo aparece más a la izquierda.

En cuanto a los países latinoamericanos, Chile es el país en donde los precios de las telecomunicaciones son muy similares a los de México, mientras que en Colombia y Argentina las telecomunicaciones en conjunto tienen precios más reducidos, por lo que se encuentran bastante más a la derecha. En el caso de Venezuela, destaca que aparezca mucho más arriba, lo cual se debe a que tiene el precio más alto (43.27 usD por Mbps) en internet de banda ancha de los 64 países, mientras que Brasil es el país con precios más altos de los países latinoamericanos considerados, por lo que se encuentra en el extremo izquierdo de la gráfica, junto con los países europeos con los precios más altos, como Eslovaquia, Francia, Suiza, Gran Bretaña y Bélgica.

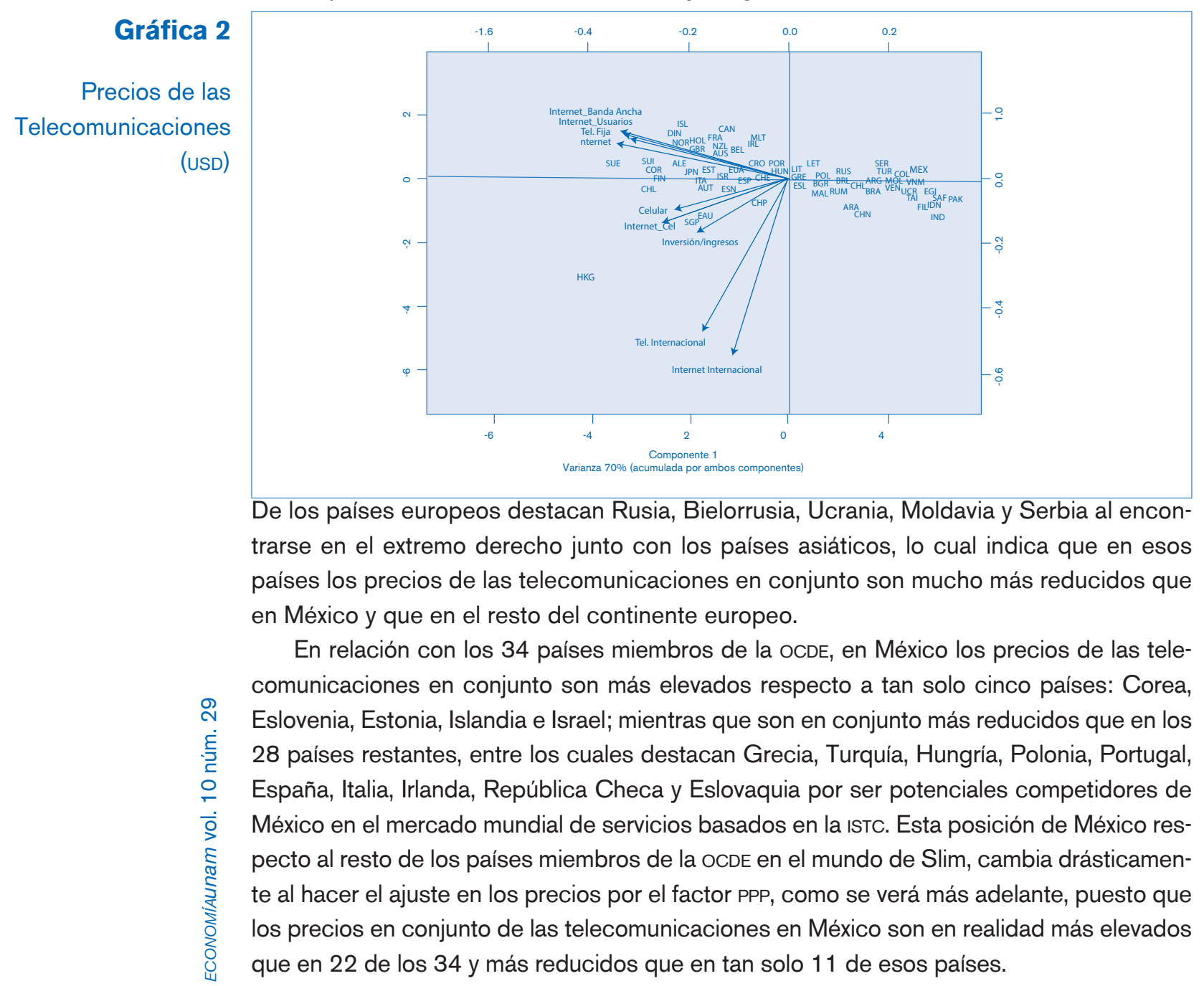


Por otra parte, en cuanto a los valores del segundo componente principal graficado en el eje " $y$ " de la misma gráfica, éstos no son tan fáciles de interpretar como los valores del primer componente principal graficado en el eje " $x$ ", debido a que el segundo componente incluye coeficientes tanto con signo positivo en las siete variables con vectores que apuntan hacia arriba, como con signo negativo en las tres variables con vectores que apuntan hacia abajo (Cel_30, Cel_100 y Cel_300). Aún así, refleja varias peculiaridades que resultan interesantes y que pueden ser confirmadas directamente con los datos.

En esta gráfica hay 54 países que aparecen más abajo o aproximadamente a la misma altura que México, esto se puede deber a cuatro circunstancias: a) en México el precio del internet de banda ancha es más alto que en esos países; $b$ ) en México el precio de la telefonía fija es más alto que en estos países; $c$ ) en México el precio de la telefonía celular es más bajo que en estos países; y d) alguna combinación de los tres incisos anteriores.

Al revisar los datos fuente a partir de los cuales se construyeron los componentes principales y sus gráficas, resulta que efectivamente en México el precio del internet de banda ancha medido en dólares corrientes es más alto que en los 54 países que aparecen más abajo, excepto Colombia, Vietnam, Pakistán, Emiratos Árabes y Bielorrusia, en los cuales el precio más alto de internet de banda ancha se compensa con precios de la telefonía fija considerablemente más bajos, lo que se expresa en su ubicación más abajo que México.

Por lo tanto, en la visión de Slim México no se encuentra tan mal posicionado en cuanto al precio del internet de banda ancha respecto a ocho de sus potenciales competidores asiáticos en el mercado mundial de servicios basados en la ISTC, por lo que se ubica más abajo respecto a Filipinas, Malasia, India, Indonesia y Arabia Saudita. Adicionalmente, Emiratos Árabes, Pakistán y Vietnam, aunque aparecen más abajo que México, tienen en realidad un precio más alto de internet de banda ancha. Aun así, entre los 54 países que se ubican por debajo o a una altura aproximadamente igual a la de México, se encuentran todos los países miembros de la OCDE, lo cual indica que el del internet precio del internet de banda ancha medido en dólares corrientes es más es más alto alto en México que en ese grupo de 34 países.

Al hacer el ajuste por el factor ppp en los precios de las telecomunicaciones, y pasar, por tanto, al mundo del poder adquisitivo real de los usuarios, la posición de México en la gráfica 3 cambia significativamente respecto a la 2. En aquélla, 48 países se encuentran a la derecha de México, lo cual indica que sus precios de las telecomunicaciones ajustados por el ppp son en conjunto más reducidos que en el país, mientras que en la gráfica 2 sólo eran treinta países.

Adicionalmente, se acentúa la posición observada en la gráfica 2 de México respecto a los países asiáticos, puesto que se encuentra muy alejado a la izquierda de ellos, lo cual indica que en esa región del mundo los precios de las telecomunicaciones en conjunto profundizan su diferencial a la baja en relación con México. Inclusive Japón, que en la gráfica 2 se ubicaba a la izquierda de México, pasa a ubicarse a su derecha.

En cuanto a los países miembros de la OCDE, resulta que en México los precios de las telecomunicaciones en conjunto son más altos que en 22 de los 34 países miembros, que se encuentran a la derecha, mientras que los precios de las telecomunicaciones son similares o más reducidos que tan sólo en 11 de estos, los cuales se encuentran a la izquierda 
o en una posición similar al país. Cabe destacar que entre los países que se encuentran a la derecha de México están Corea, Grecia, Estonia, Portugal, Irlanda, Eslovenia e Israel, los cuales, podrían servir como punto de referencia para el desarrollo de la ISTC en nuestro país. Adicionalmente, existe un conjunto de 19 potenciales competidores de México en el mercado mundial de servicios basados en la ISTC con precios de los servicios de telecomunicaciones más reducidos, como es el caso de nueve países asiáticos, China, India, Malasia, Indonesia, Singapur, Tailandia, Filipinas, Vietnam y Pakistán, además de Egipto en el continente africano, y nueve países europeos que son Rusia, Ucrania, Bielorrusia, Lituania, Letonia, Moldavia, Rumania, Chipre y Malta.

En cuanto a los países latinoamericanos, Chile continúa estando en una posición muy similar a la de México, mientras que se confirma que Argentina es el país con los precios de las telecomunicaciones que en conjunto son los más reducidos de la región. Por el contrario, continúa destacando Brasil por ser el país con los precios de las telecomunicaciones que en conjunto son los más altos.

Por su parte, la posición de México en la mitad superior de la gráfica indica que el precio del internet de banda ancha es el treceavo más alto de los 64 países considerados, aspecto en el que la posición del país no cambia mucho respecto a la gráfica 2 , en la cual también se encuentra en la mitad superior y es el dieciseisavo país con el precio más alto del internet de banda ancha de los 64 países considerados. Sin embargo, seis países asiáticos como son India, Indonesia, Malasia, Filipinas, Vietnam y Pakistán, además de Egipto en el continente africano, continúan estando bastante peor posicionados que México en cuanto al precio de internet de banda ancha, por lo que se ubican significativamente más arriba en la gráfica 3.

Completando el mundo del poder adquisitivo real de los usuarios, resulta interesante comparar la gráfica 3 con las gráficas 1.7 y 1.8 del reporte de la OCDE del pasado mes de enero (op. cit.), dado que los resultados también consideran el ajuste por el factor PPP.

\section{Gráfica 3 \\ Precios de las}

Telecomunicaciones

(PPP)

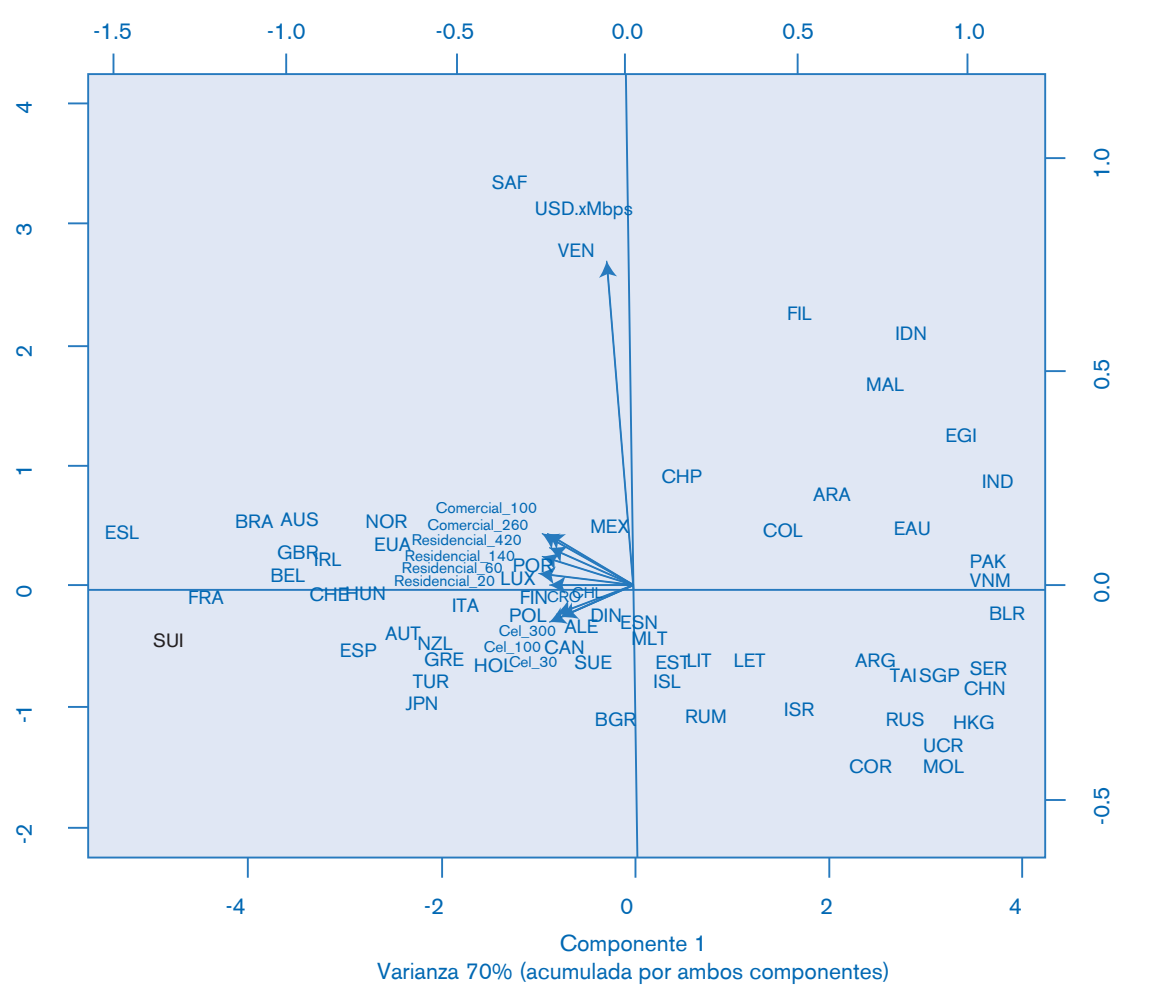


En la gráfica 1.7 aparecen la República Checa, Australia y Hungria con precios más altos que en México, en cuanto a la canasta de 140 llamadas de líneas fijas residenciales, lo que coincide con nuestra gráfica 3 para República Checa y Hungría, pero difiere en el caso de Australia, ya que ese país aparece a la derecha de México aunque no por mucho. En cuanto a la gráfica 1.8, hay siete países que tienen una canasta de 260 llamadas de telefonía comercial fija a precios más elevados que en México: República Checa, Australia, Japón, Reino Unido, Italia, Portugal y la República Eslovaca, lo que se corrobora en nuestra gráfica 3 en cuanto a Eslovaquia, Gran Bretaña y la República Checa, ya que se encuentran más a la izquierda respecto a México, mientras que en los casos de Australia, Japón, Italia y Portugal, aunque el precio de la canasta de 260 llamadas de telefonía comercial fija es mayor, los precios de las telecomunicaciones en conjunto no son tan elevados como en México, por lo que encuentran más a la derecha en la gráfica 3.

\section{México y su problemática en el mundo de las telecomunicaciones}

Lo que explica la posición de México en el mundo de la telecomunicaciones es la modalidad bajo la cual la industria se ha desarrollado, en el marco de la vía neoliberal seguida por el país.

La modalidad de desarrollo de la ISTC se caracteriza por el control monopólico de las redes, su gestión rentista y la concentración espacial de la producción y diferenciación

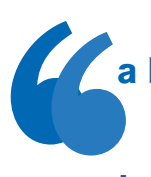

a la centralización de la producción de la industria corresponde una concentración regional de los servicios proporcionados, cuyos fundamentos son: 1) se trata de una típica industria de servicios, determinada por la dinámica de la telefonía, que se caracteriza por una alta sensibilidad al de su despliegue espacial-territorial crecimiento del resto de las actividades económicas y en cuyo crecimiento predomina, por tanto, el efecto de arrastre por la dinámica del resto de actividades; 2) existe un sobreprecio del servicio telefónico tanto fijo como móvil, y de los servicios de internet, esto último debido al amplio predominio del acceso a internet vía telefónica, lo cual expresa el control monopólico de la redes, específicamente de telefonía fija y móvil; 3) el sobreprecio de la telefonía constituye el fundamento de una sobreganancia de la rama, que, aunada a una sobrevaluación del tipo de cambio, ha financiado una vertiginosa expansión internacional del operador monopólico de la telefonía fija y móvil hacia Latinoamérica, que la ha llevado a disputarse el control del mercado latinoamericano con la empresa española Telefónica; 4) como resultado del sobreprecio de los servicios tiene lugar una baja tasa de penetración tanto de la telefonía fija como móvil, así como del servicio de internet, lo que se complementa con la baja cobertura tanto total como por habitante del ancho de banda internacional, que se traduce, en general, en un errático crecimiento de la rama de internet y la pérdida de su peso específico en la industria; 5) lo anterior se expresa en altos niveles de centralización del capital, la producción y el empleo, que se traduce en una alta concentración de los mercados de la industria en su conjunto, con el caso extremo de la telefonía donde se verifican los mayores niveles de centralización; 6) a la centralización de la producción de la industria corresponde una concentración de su despliegue espacial-territorial en la región central del país, específicamente el Distrito Federal y zona conurbada, pero con importancia relativa de la localización de empresas en el conjunto de la región norte y la región centro occidental, cuyos servicios tecnológicamente 
más avanzados (predominio o cierta importancia relativa de la telefonía, participación de relativa importancia de internet y poca presencia de las redes de cable) se concentran, igualmente, en el centro y nororiente del país, mientras los más atrasados (predominio de la telegrafía, importancia relativa de las redes de cable y poca presencia de internet) lo ha-

hace falta una clara división entre la formulación de políticas y las funciones de regulación cen en el sur y centro-norte, con regiones hibridas (predominio de la telegrafía e importancia relativa de internet) noroccidentales, centro-occidentales y orientales (Ordóñez y Bouchaín, 2011).

En consecuencia, el diagnóstico sobre la necesidad de cambios en el marco regulatorio y las recomendaciones de la OCDE deben ser planteadas con base en una consideración al enorme rezago en los niveles de penetración de los servicios y el coeficiente de inversión a ingresos, el sobreprecio de las tarifas de telefonía fija y celular, además de las de acceso a internet de banda ancha, y la reducida capacidad de integración al mercado mundial mediante la exportación de servicios intensivos en conocimiento, que caracterizan a la ISTC en México y su situación comparativa internacional, está determinada por la modalidad de desarrollo de la industria en el marco de la vía neoliberal seguida por el país.

A partir de ello coincidimos con las conclusiones del diagnóstico de la OCDE, referentes a "la falta de una clara división entre la formulación de políticas y las funciones de regulación, en conjunción con complicados procedimientos interinstitucionales [que] ha multiplicado la interposición de recursos e impugnaciones legales y ha creado confusión en el sector, lo que opone barreras a la entrada en el mercado y obstaculiza la competencia efectiva". ${ }^{17}$ Asimismo, coincidimos con las principales medidas regulatorias propuestas, a saber:

1. Garantizar bajas barreras de entrada en las telecomunicaciones, y exigir mercados impugnables donde se garanticen costos de entrada y salida bajos, que los entrantes potenciales tengan acceso a una tecnología similar, y que no existan costos hundidos; ${ }^{18}$ por lo que se deben eliminar las barreras a la inversión extranjera directa en telefonía fija y facilitarse la entrada a operadores en redes móviles.

2. La simplificación del sistema hacia la implementación de un título de concesión único (salvo en casos de recursos escasos), además de que las obligaciones deben cumplirse por parte de los operadores, garantizar la transparencia, no discriminación y la aplicación efectiva de la regulación.

3. El sistema jurídico permite suspender y en ocasiones anular las decisiones de política y regulación (como el caso del juicio de amparo), ante lo cual se pueden establecer tribunales especializados o una Corte Federal Especializada, bajo la idea de que las decisiones de la autoridad reguladora surtan efectos.

${ }^{17}$ El desempeño insatisfactorio del mercado de telecomunicaciones en México se debe en parte al uso incesante de amparos, sobre todo por parte del incumbente de telefonía fija y móvil, al cual no puede hacer frente un sistema legal disfuncional, y que ha remplazado en gran medida el derecho y la responsabilidad del gobierno de implementar las políticas y la regulación económicas. Aunque se han hecho encomiables esfuerzos tendentes a reformar los marcos de políticas y regulación, la capacidad de aplicación efectiva de las recomendaciones ha sido frustrada por los procedimientos judiciales (OCDE: 2012).

${ }^{18}$ Un costo hundido es en el que se ha incurrido independientemente de la realización del proyecto, no es relevante para la toma de decisiones, y se deben suprimir en el análisis y la evaluación de un proyecto. 
4. Eliminar la doble ventanilla que separe las funciones acerca de la formulación de políticas (ejecutivo federal), de las de regulación y supervisión (organismo regulador), éste debe de tener mayor dependencia y autonomía.

5. La autoridad reguladora debe tener la facultad de imposición de multas así como de requerir información de los agentes; la necesidad de publicar indicadose deben eliminar las barreras a la inversión extranjera directa en telefonía fija y facilitar la entrada a operadores en redes móviles res sobre la calidad de servicio y capacidad de obligar a empresas con poder de mercado, así como la necesidad de mayor transparencia en los procesos de toma de decisiones y de realizar consultas entre los operadores.

6. La COFETEL debe ser competente para identificar los cuellos de botella y necesidades de infraestructura, asimismo, debe regular las tarifas ex-ante para fomentar la competencia garantizando certeza reguladora e igualdad de condiciones de los operadores (fijar y administrar los precios tope); debe exigir al incumbente de red fija (Telmex) la consolidación de áreas de marcación local, la desagregación del bucle local en condiciones no discriminatorias y la alineación de los precios orientados a los costos, además de promover que se comparta eficientemente la infraestructura y eliminar las barreras para la obtención de derechos de paso o de vía.

7. Telmex no debería tener autorización para prestar servicios de televisión a menos de que se le aplique una fuerte regulación asimétrica, además de que la COFETEL debería estar facultada para imponer la separación funcional y estructural de cualquier operador que abuse de su poder de mercado.

la autoridad reguladora debe tener la facultad de imposición de multas así como de requerir información de los agentes
8. Se debe avanzar en la transición de la televisión digital terrestre para que se complete en 2016 y crear las condiciones para la convergencia de las telecomunicaciones y los servicios audiovisuales.

9. Se debe de licitar la fibra oscura de la CFE, respecto a lo cual coincidimos sobre la necesidad de un Plan Nacional de Banda Ancha con visión prospectiva.

Pero consideramos que este conjunto de medidas requieren de una recuperación de la capacidad de gestión y regulación del Estado no sólo sobre la ISTC, sino sobre el conjunto de la economía, que implicaría una reorientación de la vía de desarrollo del país en una perspectiva posneoliberal. En ese marco, la ISTC tendría que transitar de una industria articulada productivamente en torno a la telefonía a otra con un papel creciente de internet en la articulación del conjunto de actividades, implicando que la ISTC dejaría de ser una típica industria de servicios sensible al crecimiento del resto de la economía y tirada por ella, en favor de una industria que, sin perder su alto índice de sensibilidad, desarrollaría una importante capacidad de arrastre sobre el resto de las actividades, y, particularmente sobre las actividades intensivas en conocimiento.

En esa perspectiva, sería indispensable la promoción del desarrollo de la infraestructura en redes de fibra óptica y redes inalámbricas de $3^{\text {a }}$ y $4^{\text {a }}$ generación, lo cual implica costos de inversión considerables y crecientes que tendrían que ser afrontados mediante la fórmula que están encontrando los países europeos más avanzados en el ámbito regulatorio, como respuesta a la tendencia a la convergencia tecnológica y productiva en la industria: compartir la infraestructura entre los operadores de telecomunicaciones sobre la base de un mo- 
delo de acceso abierto, lo que constituye una estrategia que acelera el despliegue de una infraestructura nacional de banda ancha, en relación con simplemente dejarlo al libre juego de las fuerzas del mercado, especialmente en el caso de los países en desarrollo.

El Estado debería actuar, entonces, abriendo completamente el conjunto de la infraestructura básica al acceso de los competidores que proporcionan servicios hacia abajo en la cadena de valor y para los cuales la infraestructura es un insumo crucial, proceso en el cual debe mantenerse el equilibrio entre dos objetivos a menudo difíciles de compatibilizar: la preservación de un clima propicio para la inversión en infraestructura y el incremento de la competencia.

En términos instrumentales el Estado debería aprovechar que cuenta con infraestructura de redes propia (como la red de fibra oscura de la CFE y la propia red de distribución eléctrica que posibilitará acceso de banda ancha a internet en forma masiva en un futuro cercano) para concesionarla a empresas que se dediquen exclusivamente a su gestión y mantenimiento, y éstas, a su vez, la renten a empresas únicamente proveedoras de servicios intermedios a empresas o finales a consumidores, lo que evitaría el conflicto de intereses de las empresas que al mismo tiempo que son dueñas de la redes proporcionan servicios, $y$, adicionalmente, tienen que abrir sus redes a otras empresas que son sólo proveedoras de servicios y competidoras, lo que determina que la competencia se centre en las condiciones de acceso a la red y no en la innovación de los servicios que se proporcionan.

Bajo la presión competitiva de esa nueva modalidad de desarrollo del segmento de la industria basada en sus redes y con un nuevo y sólido entramado institucional, el Estado podría promover el tránsito del segmento de la industria que tiene como infraestructura a las redes privadas a una modalidad de desarrollo con esas características, y así promover el tránsito del conjunto de la industria a la nueva modalidad de desarrollo centrada en la innovación de los servicios. Una modalidad de desarrollo con tales características no sólo haría innecesario el mundo ilusorio de Slim, sino que implicaría el derrumbe del mundo real actual de la ISTC que le da cabida y del cual él mismo es promotor.

"el Estado debería aprovechar que cuenta con infraestructura de redes propia para concesionarla a empresas que se dediquen exclusivamente a su gestión y mantenimiento

\section{Anexo metodológico}

\section{Ventajas estadísticas de los componentes principales}

Los componentes principales (CP) son muy útiles para analizar los datos provenientes de " $n$ " observaciones, en nuestro caso $n=64$ países, en las cuales se llevaron a cabo las mediciones de un conjunto de " $p$ " variables, $p=9$ en la gráfica 1 y $p=10$ en las gráficas 2 y 3 . Una de las ventajas de los CP es que nos permiten evaluar el desarrollo de la ISTC en un país al considerar un conjunto de variables simultáneamente en una sola gráfica en vez de realizar gráficas por separado para cada variable.

Otra ventaja de los CP es que compensan el desarrollo de los países en algunas de las variables de telecomunicaciones con el rezago que podrían tener en otras variables, pues 
a grandes rasgos ${ }^{19}$ los CP pueden ser vistos como una especie de promedio ponderado de las variables estandarizadas ${ }^{20}$ como se ve en la siguiente fórmula:

$$
C P_{j}=\sum_{\mathrm{k}=1}^{p} a_{j k} x_{k}^{*} \text { para } \mathrm{j}=1,2, \ldots, \mathrm{p} \text { donde } \sum_{\mathrm{k}=1}^{p} a_{j k}^{2} 1
$$

Mediante el programa $\mathrm{R}^{21}$, es posible obtener tanto los CP como sus gráficas. Una de las propiedades matemáticas de los CP es la siguiente:

$$
\sum_{j=1}^{p} \operatorname{Var}\left(C P_{j}\right)=\sum_{j=1}^{p} \operatorname{Var}\left(X_{j}^{*}\right)=\sum_{j=1}^{p} 1=p
$$

Esta propiedad hace posible que al considerar tan sólo a los dos primeros componentes principales, $C P_{1}$ y $C P_{2}$, se pueda ubicar en una gráfica en dos dimensiones, con un cierto porcentaje de aproximación, ${ }^{22}$ a cada uno de los 64 países conforme a sus correspondientes valores en las "p" variables medidas originalmente. En dicha gráfica además se incluye un vector o flecha cuyas coordenadas son los coeficientes $\left(a_{1 k}, a_{2 k}\right)$ por cada una de las variables $X_{k}^{*}$.

\section{Selección de países y medición del precio del internet de banda ancha}

La Unión Internacional de Telecomunicaciones ${ }^{23}$ (UIT) es el organismo especializado de las Naciones Unidas para las tecnologías de la información y la comunicación, el cual recopila y publica, entre otros resultados, datos de 161 variables del sector telecomunicaciones en 212 países que van desde el año 1960 al año 2010.

Una variable crucial en el desarrollo de las telecomunicaciones de un país es el costo del internet medido en USD por MBPS (megabits por segundo), pero al inspeccionar las variables reportadas por la UIT no encontramos una que reflejara correctamente el costo del internet de banda ancha pues es muy frecuente que la velocidad de la conexión sea menor a la velocidad anunciada por el proveedor, por lo que recurrimos a otra fuente de información: netindex ${ }^{24}$ en cuyo sitio de internet los usuarios de este servicio pueden medir la velocidad real de su conexión. Debido que en netindex sólo hay mediciones para 64 países para el año 2012, la presente comparación internacional la realizamos con base en estos, los cuales enlistamos a continuación:

${ }^{19}$ Para una descripción más detallada del análisis de componentes principales sugerimos consultar el siguiente texto: Multivariate statistical methods: a primer, Bryan F.J. Manly, Chapman \& Hall, 3rd. edition, 2005, pp. 75-86.

$20 \dot{x_{k}}=\frac{x_{k}-x_{k}}{\sqrt{\frac{\sum_{k=1}^{n}\left(x_{k}-x_{k}\right)^{2}}{n}}}$, esto permite incluir simultáneamente variables con unidades de medición muy diversas

${ }^{21} \mathrm{R}$ : A language and environment for statistical computing. R Foundation for Statistical Computing, Vienna, Austria. ISBN 3-900051-07-0, URL http://www.R-project.org, R Development Core Team (2011)

${ }^{23}$ ie varianza explicado en la gráfica $=\frac{\operatorname{var}\left(C P_{1}\right)+\operatorname{var}\left(C P_{2}\right)-2 \mid \text { co } \operatorname{var} i \operatorname{anzavar}\left(C P_{1}\right)+\operatorname{var}\left(C P_{2}\right)}{p}$

${ }^{24} \mathrm{http}: / /$ www.netindex.com/value/allcountries/. Consulta de COST PER MBPS realizada el mes de marzo de 2012. 
- 8 países en América: Argentina (ARG), Brasil (BRA), Canadá (CAN), Chile (CHL), Colombia (CoL), México (MEX), Estados Unidos (EU) y Venezuela (VEN).

- 37 países en Europa: Alemania (ALE), Austria (AUt), Bélgica (BeL), Bulgaria (BGR), Bielorrusia (BLR), Chipre (CHP), República Checa (CHE), Dinamarca (DIN), Eslovaquia (ESL), Eslovenia (ESN), España (ESP), Estonia (EST), Finlandia (FIN), Francia (FRA), Gran Bretaña (GBR), Grecia (GRE), Croacia (CRO), Holanda (HOL), Hungría (HUN), Irlanda (IRL), Islandia (ISL), Italia (ITA), Lituania (LIT), Luxemburgo (LUX), Letonia (LET), Moldavia (MOL), Malta (MLT), Noruega (NOR), Polonia (POL), Portugal (POR), Rumania (RUM), Rusia (RUS), Serbia (SER), Suecia (SUE), Suiza (SUI), Turquía (TUR) y Ucrania (UCR).

- 15 países en Asia: ${ }^{25}$ Arabia Saudita (ARA), Corea del sur (COR), China (CHN), Emiratos Árabes Unidos (EAU), Filipinas (FIL), Hong Kong (HKG), Indonesia (IDN), India (IND), Israel (ISR), Japón (JPN), Malasia (MAL), Pakistán (PAK), Singapur (SGP), Tailandia (TAI) y Vietnam (VNM).

- 2 países en Oceanía: Australia (AUs) y Nueva Zelanda (NZL).

- 2 países en África: Egipto (EGI) y Sudáfrica (SAF).

\section{Selección de las variables y del año de observación}

Seleccionamos el año 2008 para realizar la comparación internacional con el fin de que los datos no resultaran afectados de ninguna manera por la crisis económica mundial que si bien tuvo lugar en 2007-2009, sus efectos no se manifestaron sino hasta este último año.

Para la selección de las variables a incluir en el análisis, consideramos las variables reportadas por la uाT que tuvieran datos para el año $2008^{26}$ en la totalidad de los 64 países enlistados en la sección anterior, de tal manera que quedaron las siguientes 29 variables, además del costo del internet de banda ancha reportado por netindex para el año 2012:

\section{a) 6 variables con los niveles de cobertura}

Descripción por la UIT
Fixed telephone lines per 100 inhabitants
Mobile cellular subscriptions per 100 inhabitants
Mobile cellular subscriptions with access
to data communication at broadband speed per 100 inhabitants.
Internet subscriptions per 100 inhabitants
Fixed broadband subscriptions per 100 inhabitants
Internet users (\%)

Descripción por la UIT Internet users (\%)

\author{
Abreviatura en gráficas \\ Tel. Fija \\ Celular \\ Internet_Cel \\ Internet \\ Internet_Banda Ancha \\ Internet_Usuarios
}

b) 18 tarifas en USD de telefonía local tanto fija como móvil, con las cuales obtuvimos los precios de las nueve canastas descritas en la siguiente sección.

${ }^{25}$ Aunque Hong Kong es parte de China lo consideramos como si fuera un país aparte pues los datos de netindex y de la UIT se encuentran reportados por separado.

${ }^{26}$ Debido a que uno de los requisitos matemáticos del análisis de componentes principales es que no falte ningún dato, cuando no estaba disponible el dato para el año 2008 , consideramos los datos reportados por la UIT para años anteriores. Con este procedimiento $90 \%$ de los datos corresponden a 2008 y el restante 10\% corresponde a 2005-2007. En el caso de México, 100\% de los datos corresponden a 2008. 
c) 2 variables que se refieren al nivel de inversión y de ingresos en telecomunicaciones, a partir de las cuales calculamos el siguiente indicador:

$$
\frac{\text { Inversión }}{\text { Ingresos }}=\frac{\sum_{t=1990}^{2008} \frac{\text { Total annual investment in telecomunication (US\$) }}{\text { Total revenue from all telecommunication services (US\$) }}}{19}
$$

Y finalmente, otras tres variables que se refieren a la capacidad de integración al mercado mundial de servicios basados en la ISTC, a partir de las cuales calculamos los siguientes dos indicadores. ${ }^{27}$

\section{Medición de los precios de las telecomunicaciones}

En cuanto a los precios de la telefonía, calculamos los precios de seis canastas de telefonía fija y de tres canastas de telefonía celular con base en la metodología aplicada por la OCDE: $:^{28}$

\begin{tabular}{|l|l|}
\hline \multicolumn{1}{|c|}{ Canasta OCDE } & \multicolumn{1}{c|}{ Abreviatura en gráficas } \\
\hline Residential 20 calls per month basket & Residencial_20 \\
\hline Residential 60 calls per month basket & Residencial_60 \\
\hline Residential 140 calls per month basket & Residencial_140 \\
\hline Residential 420 calls per month basket & Residencial_420 \\
\hline Business 100 calls per month basket & Comercial_100 \\
Business 260 calls per month basket & Comercial_260 \\
\hline Mobile 30 calls per month basket & Cel_30 \\
\hline Mobile 100 calls per month basket & Cel_100 \\
\hline Mobile 300 calls per month basket & Cel_300 \\
\hline
\end{tabular}

Cabe mencionar que solo fue posible realizar la medición de los precios de estas canastas de telefonía a nivel local pues, por los motivos expuestos en la sección anterior, solo disponemos de 18 tarifas a este nivel geográfico para la totalidad de los 64 países incluidos en la presente comparación.

Por otra parte, para ajustar los precios de las telecomunicaciones por el factor "Purchasing Power Parity" (PPP) utilizamos los índices reportados por el banco mundial ${ }^{29}$ en el año 2008 para hacer el ajuste en el caso de las canastas de precios de telefonía tanto fija como móvil; y los reportados para el año 2010, que es el más reciente disponible, para hacer el ajuste en el caso de los precios de internet reportados por netindex. La fórmula

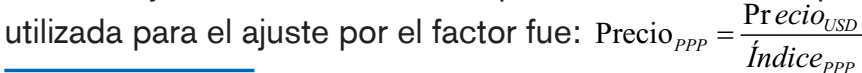

${ }^{27}$ En cuanto al superávit en el tráfico telefónico internacional, consideramos que el saldo neto reportado en la balanza de pagos por el fondo monetario internacional correspondiente al concepto de servicios de telecomunicaciones sería un mejor indicador de la capacidad de integración al mercado mundial de servicios basados en la ISTC pero no fue posible utilizarlo debido a que este dato solo se encuentra disponible en la página http://www.imfstatistics.org/ para 42 de los 64 países reportados por netindex.

${ }^{28} \mathrm{http}: / /$ www.oecd.org/dataoecd/44/29/48242089.pdf

${ }^{29}$ http://data.worldbank.org/indicator/PA.NUS.PPPC.RF. Consultada el mes de marzo del presente año. 


\section{Bibliografía}

Dabat, A. y Ordóñez, S., Revolución informática, nuevo ciclo industrial e industria electrónica en México,

México, Distrito Federal, IIEC-UNAM-Casa Juan Pablos, 2009

Hausman, Jerry y Agustín Ros, Corrigiendo la valoración errónea de la OCDE sobre la competencia de las telecomunicaciones en México, 2012.

http://data.worldbank.org/indicator/PA.NUS.PPPC.RF.

http://dx.doi.org/10.1787/9789264166790-es.

http://www.imf.org.

http://www.imfstatistics.org/.

http://www.itu.int.

http://www.netindex.com/value/allcountries/.

http://www.oecd.org/dataoecd/44/29/48242089.pdf.

Manly, Bryan F.J., Chapman \& Hall, Multivariate statistical methods: a primer , 3rd. edition, 2005.

OCDE, Estudio de la OCDE sobre políticas y regulación de telecomunicaciones en México, 2012.

Ordóñez, Sergio, Nueva fase de desarrollo y capitalismo del conocimiento: elementos teóricos, en

Comercio Exterior, vol. 54, num. 1, enero, 2004.

Ordóñez Sergio, Bouchaín Rafael, Capitalismo del conocimiento e industria de servicios de telecomunicación en México, IIEC-UNAM, 2011.

Ordóñez, Sergio, "Crisis global y procesos de innovación de base electrónica-informática en América

Latina", en del Valle, Carmen (Coord), El pensamiento Latinoamericano sobre el cambio tecnológico para el desarrollo, IIEC-UNAM, México, Distrito Federal, en prensa.

Sidak, Gregory, La propuesta de la OCDE por cartelizar las telecomunicaciones mexicanas, 2012. 